\title{
Pharmacovigilance for Biosimilars
}

\section{Maria I Karampola ${ }^{1,2}{ }^{*}$ and Christos E Emmanouilides ${ }^{1}$}

${ }^{1}$ Interbalkan Medical Center, Department of Oncology, Asklipiou 10, Pylaia, 57001, Greece

${ }^{2}$ Oncologic Office, D.Gounari 35, Thessaloniki, 54622, Greece

*Corresponding author: Maria I Karampola, El. Venizelou 26, Chalastra, Thessaloniki, Post Code: 57 300, Greece, Tel: 306976157798; E-mail: maria.karampola@gmail.com

Received date: February 03, 2016; Accepted date: February 17, 2016; Published date: February 22, 2016

Copyright: (c) 2016 Karampola MI, et al. This is an open-access article distributed under the terms of the Creative Commons Attribution License, which permits unrestricted use, distribution, and reproduction in any medium, provided the original author and source are credited.

\begin{abstract}
Pharmacovigilance can be defined as the process of collection of safety and to a lesser extent efficacy information regarding pharmaceuticals that have already been launched in the market. It is possible that all toxicity has not been precisely identified before drugs obtain approval for marketing. In addition to relying in ad hoc toxicity reporting, formal studies may be conducted after drug approval to confirm safety (post-authorization safety study, PASS), as well as efficacy (post-authorization efficacy study, PAES). These studies are of paramount importance for biosimilars, due to their macromolecular and potential immunogenic nature. Biosimilars are used in many medical fields, including oncology and rheumatology.

In order to guarantee the success of post-authorization studies, both health care professionals and patients are required to contribute by reporting adverse drug reactions (ADR). The European Union even urges patients to directly report ADR. Apart from physicians, pharmacists play an important role in pharmacovigilance, by keeping accurate records enabling the identification of a specific batch of a biosimilar to be linked to a particular ADR. Thus the principle of non-inter changeability of the prescribed product ought to be followed. PASS are mandatory for biosimilars at the responsibility of the market authorization holder. Implementation of rigorous pharmacovigilance policies will promote physicians' confidence in them, hopefully resulting in financial alleviation of Health Systems.
\end{abstract}

Keywords Pharmacovigilance; Biosimilars; Adverse drug reactions; Drug safety

\section{Introduction}

Pharmacovigilance is the practice of monitoring the effects of medical drugs after they have been licensed for use, especially in order to detect previously unreported adverse effects. Although drugs pass rigorous testing before authorization, it is possible that uncommon toxicities evaded registrational trials. The compelling need for introducing new therapeutics forces regulatory authorities to approve drugs when reasonably adequate safety profile has been determined. It would have been unreasonable and impractical to strive for accurate assessment of rare toxicities before approval, because the required clinical trials would have been large, lengthy and costly. Therefore, pharmacovigilance is of paramount importance to exclude such a possibility for an already marketed drug. Observations during pharmacovigilance period have better defined the toxicity profile of many drugs and in certain occasions resulted in the revocation of drug licenses.

Pharmacovigilance is primarily based on reports of adverse drug reactions (ADR) by physicians. However, it has been noticed that underreporting is a serious issue that does not allow drug safety to be precisely characterized. In a study looking into the causes of underreporting, lack of sufficient medical history information was found in $44 \%$ of the cases, time restriction in $70 \%$ as well as difficulty to relate the ADR with the specific drug in $81 \%$ [1]. Health care professionals (HCP) suggested that more awareness regarding pharmacovigilance was needed, as well as sensitization about the impact of ADR on patient safety; promotion of a new tool for reporting, making the process easy and less time consuming, would encourage HCP to provide information through this platform [2].

The European Union also empowered patients to directly report ADR to authorities. Such reporting is complementary to physicians' reporting and to a certain extent could make up for the underreporting by HCP. Patients often provide information related to ADR occurring in the community setting [3]. Patients tend to report not serious or already known ADR [3,4], however the reporting appears to be similar between patients and HCP [5,6]. Finally the European Medicine Agency (EMA) has created the following web site: www.adrreports.eu, where HCP can report ADR which are expected to be evaluated [2].

In addition to ad hoc reports of ADR, regulatory authorities can impose the implementation of formal post-authorization safety studies (PASS) to be conducted by the marketing authorization holder (MAH), usually the pharmaceutical company that markets the drug. PASS are studies whereby, characterization, identification or quantitation of a safety hazard as well as the overall safety of the product is assessed after having been launched into the market.

PASS are governed by well-defined regulation of the European Union (Directive 2001/83/EC, (DIR) Art 1 [7]. It is a structured process of predetermined sample size, with specific aims and research questions. PASS are usually non-interventional studies as the drugs are used according to their indications. Results are closely monitored by the marketing authorization holder and are communicated to the national and international regulatory bodies. The captured events should be assessed in terms of severity and effect on the risk/benefit ratio of the drug. Aggregated data are analyzed centrally in order to determine whether the observed risks deter clinical usefulness. 
Page 2 of 3

We discuss below the importance of a thorough pharmacovigilance program after market authorization pertaining to biosimilar agents.

\section{What is a Biosimilar?}

A new generation of drugs, called biological drugs or biologics, have been introduced in many medical fields, such as oncology, hematology rheumatology and others. These drugs are polypeptides or monoclonal antibodies; they are produced by genetic recombination techniques in living cells and thereafter purified by complex procedures. Biosimilars are biologic drugs produced by a different company aiming at imitating the original product [8]. Biosimilars have the same peptide sequence, but may not be identical due to post-trascriptional modification or alterations during the purification process. The approximation of biosimilars to the prototype is an area of concern for physicians [9] and therefore there is legislation and procedures to be followed after their market launch [10].

The main difference between biosimilars and generics is that the former are produced in bacteria or other living cells by recombinant DNA techniques, while the later are chemically synthesized in the laboratory. Generics contain the exactly same active agent as the initial drug, while biosimilars are variants of their original biological countrpart [11]. The production process of biosimilars is inherently complicated. As the final product (protein or macromolecule) is affected by glycosylation, isolation and purification methodology, it is impossible for a biosimilar to be identical to the originator. For that reason, in contrast to generics, biosimilars are required to undergo a clinical comparability trial against the prototype drug, to ensure there is clinical similarity between them. Thus, the main purpose of such a study is to prove similarity and not to benefit patients; in this sense, such studies are a novelty in therapeutics [12]. Studies designed for biosimilars are blinded randomized phase 3 [13]. Such comparability studies are required to demonstrate equivalence with the prototype in one clinical setting and in at least one clinical outcome, usually the response rate. If equivalence is demonstrated, the biosimilar is allowed to enter the market, for all the indications of the original drug. Thus, biosimilars are tested only in a few hundred patients of the one arm of a phase 3 study prior to market authorization. This is a reason for concern, as the probability of a rare but serious side-effect cannot be excluded by such studies.

Pharmaceutical companies produce biosimilars by using different cell lines as well as different manufacturing processes resulting in differences of tertiary and quaternary structure, with a putative effect on immunogenicity. Other factors affecting immunogenicity are the medical history of the patient, other drugs used at the same time, as well as host factors such as age, sex, fitness [14]. The route of administration also plays an important role in immunogenicity; for instance, in the case of trastuzumab given subcutaneously, immunogenicity was assessed prior to approval [15]. A well-known case of a harmful biosimilar is the erythropoietin biosimilar Eprex which caused red cell aplasia [16] when administered subcutaneously. The examples given prove the need for long term surveillance of biosimilars after having been available in the market.

In oncology, biologic drugs may increase the survival rate of patients. This is the case of rituximab for aggressive B-cell lymphomas and trastuzumab in the adjuvant treatment of early stage c-erbB2 overexpressing breast cancer. If immunogenicity issues are not cleared for the corresponding biosimilars, physicians may hesitate to use them in this type of potentially curable patients.

\section{Pharmacovigilance}

The pharmaceutical industry is required to support an active pharmacovigilance program for biosimilars that includes both a PASS and a PAES (post authorization efficacy study). To fulfil the task, a trained and qualified person of the pharmaceutical company must be in charge of supervising the procedures as well as all relevant logistics. $\mathrm{EU}$ has pharmacovigilance legislation for both the above types of studies under the Directive 2010/84/EU [12]. Market authorization holders are required to report immediately and in aggregate any important findings of the PASS to the national and international authorities. Both the company and the authorities have the responsibility to raise alarms, if needed, and to make decisions regarding the safety of the product. PASS and PAES studies are carefully designed under the guidance of the authorities in order to cover all the concerns and maximize the safety of the public.

The ability to track each biomedical medicinal product is closely related to pharmacovigilance. It is recommended to discourage biosimilar interchangeability by physicians or pharmacists. Ideally, a certain patient should always receive the same biosimilar product. Since the medical community is primarily concerned about the safety of biosimilars, medical systems ought to enable physicians to continue the same brand biosimilar for every individual patient. Substitution by the doctor or automatic substitution by the pharmacy must be avoided. This is the only way to accumulate reliable safety information for new biosimilars. It is very important for payors and central pharmacists to realize that biosimilars are different from generics in that aspect and to develop different set of rules for procurement, registering and dispensing biosimilars.

These issues underline the need for certain degree of freedom for physicians in decision making about the therapeutic use of biosimilars. Payors and regulators may be allowed to provide a general guidance or declare a preference, but ultimately the physician's choice ought to be respected, especially if supported by a medical justification. For instance, it may not be appropriate to readily implement the use of new biosimilars in curable patients, as explained above. Also, changing to a biosimilar in the middle of a therapy should not be encouraged, mainly for pharmacovigilance issues. Proper education of the involved professionals will help elucidate the peculiarities of biosimilars and the rationale for the concepts mentioned above. The Alliance for safe Biologic Medicines and the European Association for Bio-industries carried out a research, the conclusions of which stated that clinicians would prefer their patients to remain on the initial biosimilar prescribed and not to be given a similar drug either by a colleague or the pharmacist [17]. Maintaining accurate records in the clinic but also at the dispensing pharmacy is of paramount importance. The use of INN (International Non-proprietary Name) for each different biosimilar has been proposed and, if implemented, would facilitate the identification of differences between original drugs and biosimilars. Drug records including the batch number, the INN as well as the brand name kept by the pharmacists would greatly contribute to the pharmacovigilance program of biosimilars [14].

Therefore, a rigorous pharmacovigilance program, incorporating PASS and supported by all health professionals involved, would eventually reassure the community regarding their safety. Active engagement of health systems and even patients would also be required. Biosimilars would then be in the position to hopefully achieve their goal, which is cost containment through regulation or competition, enabling the reallocation of funds to other public health areas. 


\section{References}

1. Steurbaut S, Hanssens Y (2014) Pharmacovigilance: empowering healthcare professionals and patients. Int J Clin Pharm 36: 859-862.

2. European medicines agency (2012) Pharmacovigilance legislation.

3. Leone R, Moretti U, D'Incau P, Conforti A, Magro L, et al. (2013) Effect of pharmacist involvement on patient reporting of adverse drug reactions: first Italian study. Drug Saf 36: 267-276.

4. Marques J, Ribeiro-Vaz I, Pereira AC, Polonia J (2014) A survey of spontaneous reporting of adverse drug reactions in 10 years of activity in a pharmacovigilance centre in Portugal. Int J Pharm Pract 22: 275-282.

5. Inch J, Watson MC, Anakwe-Umeh S (2012) Patient versus healthcare professional spontaneous adverse drug reaction reporting: a systematic review. Drug Saf 35: 807-818.

6. Avery AJ, Anderson C, Bond CM, Fortnum H, Gifford A, et al. (2011) Evaluation of patient reporting of adverse drug reactions to the UK 'Yellow Card Scheme': literature review, descriptive and qualitative analyses, and questionnaire surveys. Health Technol Assess 15: 1-234.

7. European Medicines Agency (2016) Guidelines on good pharmacovigilance Practice.

8. Emmanouilides CE, Karampola MI, Beredima M (2015) Biosimilars: Hope and concern. J Oncol Pharm Pract.
9. Misra M (2012) Biosimilars: current perspectives and future implications. Indian J Pharmacol 44: 12-14.

10. European Medicinal Agency (2013) Guideline on similar biological medicinal products.

11. Sekhon BS, Saluja V (2011) Biosimilars: an overview. Biosimilars 1: 1-11.

12. European Commission (2013) A consensus information document. What you need to know about Biosimilar Medicinal Products.

13. Jeske W, Walenga JM, Hoppensteadt D, Fareed J (2013) Update on the safety and bioequivalence of biosimilars - focus on enoxaparin. Drug Healthc Patient Saf 5: 133-141.

14. Niederwieser D, Schmitz S (2011) Biosimilar agents in oncology/ haematology: from approval to practice. Eur J Haematol 86: 277-288.

15. Ismael G, Hegg R, Muehlbauer S, Heinzmann D, Lum B et al. (2012) Subcutaneous versus intravenous administration of (neo) adjuvant trastuzumab in patients with HER2-positive, clinical stage I-II breast cancer (HannaH study): a phase 3, open-label, multicentre, randomized trial. Lancet Oncology 13: 869-878.

16. Choy E, Jacobs IA (2014) Biosimilar safety considerations in clinical practice. Semin Oncol 1: S3-S14.

17. EuropaBio, SafeBiologics (2013) ASBM European physician's survey on biosimilars: key findings on knowledge, naming, traceability and physicians' choice. 